\title{
MAPS OF STIEFEL MANIFOLDS AND A BORSUK-ULAM THEOREM
}

\author{
by JAN JAWOROWSKI
}

(Received 29th January 1988)

\section{Introduction}

We are concerned with the following classical version of the Borsuk-Ulam theorem: Let $f: S^{n} \rightarrow R^{k}$ be a map and let $A_{f}=\left\{x \in S^{n} \mid f x=f(-x)\right\}$. Then, if $k \leqq n, A_{f} \neq \phi$. In fact, theorems due to Yang [17] give an estimation of the size of $A_{f}$ in terms of the cohomology index. This classical theorem concerns the antipodal action of the group $G=\mathbb{Z}_{2}$ on $S^{n}$. It has been generalized and extended in many ways (see a comprehensive expository article by Steinlein [16]). This author $([9,10)]$ and Nakaoka [14] proved "continuous" or "parameterized" versions of the theorem. Analogous theorems for actions of the groups $G=S^{1}$ or $S^{3}$ have been proved in [11], and [12]; compare also $[4,5,6]$.

A tool in estimating the size of the set $A_{f}$ (for $G=\mathbb{Z}_{2}$ ) in terms of index is the first Stiefel-Whitney class of a space with a free involution. Similarly, such an estimate for $G=S^{1}$ makes use of the first Chern class; and for $G=S^{3}$ the first Pontriagin class is used. A natural question arises of whether there exists a corresponding result using other characteristic classes.

Various extensions of the concept of index were defined and used by Fadell and Husseini (see [5, 6]). In a forthcoming paper [7], Fadell and Husseini introduce a general notion of index for an arbitrary compact Lie group action as an ideal-valued function. I arrived independently at the concept of an ideal-valued index and presented my results, with an application to a geometric situation, at the NATO Advanced Study Institute on "Variational Methods in Nonlinear Problems" held in Montreal in July 1986, where Fadell presented his joint results with Husseini; this is how I first learned about their recent work. I understand, however, that Fadell and Husseini defined their general notion of index before me and I am pleased to acknowledge their priority in developing the index theory. In fact, a suggestion that the index can be defined as an ideal is mentioned in Remark (3.5) of [5]. I shall use the notation Ind ${ }^{G}$ for the $G$-index introduced by Fadell and Husseini and prove some properties of Ind $^{G}$ (Proposition (3.3) and Theorem (3.4)) which will be needed in this paper.

In theorems of the Borsuk-Ulam type for a general compact Lie group $G$ we usually consider a map $f: X \rightarrow W$ of a $G$-space $W$, for instance, into a representation space for $G$; and we try to estimate the size of the set $A_{f}$ where the $G$-symmetry becomes degenerate under $f$. The degeneracy set may be defined in various ways depending on the context. 
For instance, if $f: X \rightarrow W$ is an equivariant map of $X$ into a representation space $W$, we may want $A_{f}$ to be the set of zeros of $f$. More generally, for any invariant subset $\tilde{W}$ of $W$, we can set $A_{f}(\tilde{W}):=f^{-1} \tilde{W}$. If we don't want to start necessarily with an equivariant map $f: X \rightarrow W$, we can apply the averaging construction, replace $f$ by its average $\operatorname{Av} f: X \rightarrow W$ and define $A_{f}(\tilde{W}):=(\operatorname{Av} f)^{-1} \tilde{W}$. (Compare [11] and [12]). The classical Borsuk-Ulam theorem asserts that for any map $f: S^{n} \rightarrow \mathbb{R}^{n}$ there is a point in $S^{n}$ where the average of $f$ (with respect to the antipodal actions on the source space and on the target space) is zero.

1.1. Example. The following example is a direct generalization of the Borsuk-UlamYang situation of $f: S^{n} \rightarrow \mathbb{R}^{k+1}$ from the group $G=\mathbb{Z}_{2}(O(1))$ to $G=O(m)$ :

Let $X$ be the Stiefel manifold $V_{m}\left(\mathbb{R}^{m+n}\right)$ of orthonormal $m$-frames in $\mathbb{R}^{m+n}$ and let $f: X=V_{m}\left(\mathbb{R}^{m+n}\right) \rightarrow\left(\mathbb{R}^{m+k}\right)^{m}=W$ (be a map. In other words, $f$ assigns to every $m$-frame in $V_{m}\left(\mathbb{R}^{m+n}\right)$ an $m$-tuple of vectors in $\mathbb{R}^{m+k}$. Let $\tilde{W}$ be the subset of $W$ consisting of the $m$-tuples which are not linearly independent. We are asking about the size of $A_{f}=(\operatorname{Av} f)^{-1} \tilde{W}$; i.e., $A_{f}$ is the degeneracy set in our example. Here the group $G=O(m)$ acts on $V_{m}\left(\mathbb{R}^{m+n}\right)$ and on $\left(\mathbb{R}^{m+k}\right)^{m}$ in the standard way: Thus if $w \in W$, then $w$ can be thought of as an $(m+k) \times m$ matrix (having $m+k$ rows and $m$ columns). For $g \in O(m)$ we define $g w:=w \cdot g$, where the dot is the matrix multiplication. Then the action is free in $W-\tilde{W}$ : if $w \in W-\tilde{W}$, i.e., Rank $w=m$, and $w \cdot g=w$, there is an $(m \times m)$-submatrix $A$ of $w$ which is invertible. Then $A \cdot g=A$ and thus $g$ is the identity. The converse is also true: if $w \in W$ and Rank $w<m$, then one can find a matrix $g \in O(m)$ other than the identity such that $w \cdot g=w$. Of course, if $m=1$, then $X=S^{n}, W=\mathbb{R}^{k+1}$, and we are in the BorsukUlam-Yang situation. We will prove a theorem in which the size of $A_{f}$ is described in terms of index, in a way similar to the assertion of the Borsuk-Ulam theorem. An estimate of the size of $A_{f}$ will be given in terms of cohomology, but, as a corollary we will find a lower bound for the covering dimension, $\operatorname{dim} A_{f}$, of $A_{f}$ : In the case $m=2$, we will show that $\operatorname{dim} A_{f} \geqq 2 n-k-1$; if, in addition, $k=n-1$ and $n=2^{s}-1$, then $\operatorname{dim} A_{f} \geqq$ $n+1$.

There exist also "continuous" versions of the results proved here, for spaces and maps over a base space. They are analogous to those of $[9,10,12]$. We shall deal with them in a future paper.

\section{Index}

Let $G$ be a compact Lie group. We shall be using the Alexander-Spanier cohomology with coefficients in $\mathbb{Z}_{2}$ (which will be suppressed from the notation) and the Borel equivariant cohomology. If $X$ is a $G$-space then $X_{G}:=E G_{G} \times X$ where $E G$ is a universal space for $G, G$ acts on $E G \times X$ by $g(e, x)=(g e, g x)$ and $E G_{G} \times X:=(E G \times X) / G$. The map $X_{G} \rightarrow(E G) / G=B G$ induced by the first projection $E G \times X \rightarrow E G$ is a bundle with fibre $X$. If $G$ acts trivially on $X$ then $X_{G} \cong B G \times X$.

The equivariant cohomology of $X$ is $H_{G}^{*} X:=H^{*} X_{G}$. If $G$ acts freely on $X$ then the map $X_{G} \rightarrow X / G$ induced by the second projection $E G \times X \rightarrow X$ is a bundle with a contractible fibre $E G$; hence $H_{G}^{*} X \cong H^{*}(X / G)$. 
If $(*)$ denotes a one-point space then the constant map $E G \rightarrow(*)$ induces an isomorphism $H_{G}^{*}(*) \cong H_{G}^{*} E G$. We will be identifying the groups $H_{G}^{*}(*), H_{G}^{*} E G$ and $H^{*} B G$ under this isomorphism.

Proposition (compare $[11,(5.1)])$ 2.1. Let $X$ be a free $G$-space, let $\varphi: X \rightarrow E G$ be an equivariant map and let $c=c_{X}: X \rightarrow(*)$ be the constant map. Then under the identification $H_{G}^{*} E G=H_{G}^{*}(*)=H^{*} B G$ and $H_{G}^{*} X=H^{*}(X / G)$ we have $\varphi^{*}=c^{*}: H^{*} B G \rightarrow H^{*}(X / G)$.

Definition 2.2. Let $G$ be a compact Lie group and let $X$ be a $G$-space. Then the $G$ index of $X$ is defined to be the kernel of the $G$-cohomology homomorphism induced by the constant map $c_{X}: X \rightarrow(*)$;

$$
\operatorname{Ind}^{G} X:=\operatorname{Ker}\left(c^{*}: H_{G}^{*}(*) \rightarrow H_{G}^{*} X\right)
$$

Thus the index of $X$ is an ideal in the $G$-cohomology ring of a point.

In the classical case, when $G=\mathbb{Z}_{2}$ is acting freely on $X$, the non-trivial element of $\mathbb{Z}_{2}$ represents a free involution on $X$. In the case $B G \cong \mathbb{R} P^{\infty}, H_{G}^{*}(*) \cong H^{*} \mathbb{R} P^{\infty}$ is a polynomial algebra over $\mathbb{Z}_{2}$ on one generator in dimension one, the first Stiefel-Whitney class $w_{1} \in H_{Z_{2}}^{1}(*) \cong H^{1} \mathbb{R} P^{\infty}$. Its image under $c^{*}$ in $H_{\mathbf{Z}_{2}}^{1} X=H^{1}\left(X / \mathbb{Z}_{2}\right)$ is the characteristic class of the involution, $w_{1}(X)=c^{*} w_{1}$. The kernel of $c^{*}$ is the ideal generated by $w_{1}^{n+1}$, for some integer $n$, and thus $\operatorname{Ind}^{\mathrm{Z}}(X)$ can be identified with that integer; it is the largest integer $n$ such that $w_{1}^{n}(X) \neq 0$. This corresponds to the classical definition of index of space with a free involution. In an analogous way, for free actions of $G=S^{1}$ and $G=S^{3}$ (and for cohomology with rational coefficients), the index can also be identified with an integer (compare $[4,5,6,11,12]$ ).

That the index is natural is expressed by the following proposition.

Proposition 2.3. Let $X$ and $Y$ by $G$-spaces and let $f: X \rightarrow Y$ be an equivariant map. Then

$$
\operatorname{Ind}^{G} Y \subset \operatorname{Ind}^{G} X \text {. }
$$

The proof is immediate.

The following theorem is a general principle of which the classical Borsuk-UlamYang theorem is a special case (compare Remark (5.2)).

Theorem 2.4. Let $X$ and $W$ be $G$-spaces and assume that $X$ is paracompact. Let $f: X \rightarrow W$ be an equivariant map, and let $\tilde{W}$ be a closed invariant subset of $W$. Then

$$
\left(\operatorname{Ind}^{G} f^{-1} \tilde{W}\right) \cdot\left(\operatorname{Ind}^{G}(W-\tilde{W})\right) \subset \operatorname{Ind}^{G} X .
$$

Remark. The proof is analogous to the proof of part (b) of Proposition (2.8) of [6] (additivity property of the integer-valued index). Compare also [9, p. 113], [10, p. 160], $[11$, p. 161] and [12, p. 148]. Thus (2.4) expresses a crucial principle used in these proofs. 
Proof. As before, for every space $Y, c=c_{Y}: Y \rightarrow(*)$ is the constant map of $Y$ into a one point space. Let $A_{f}=f^{-1} \tilde{W}$ (thus $A_{f}$ corresponds to the singularity set in the Borsuk-Ulam-Yang situation) and let $a \in \operatorname{Ind}^{G} A_{f}$; that is, $c_{A_{f}}^{*} a=0$. Consider $c_{X}^{*} a \in H_{G}^{*} X$. Thus $\left(c_{X}^{*} a\right) \mid A_{f}=0$. By the continuity of $H_{G}^{*}$ it follows that there exists a neighbourhood $N$ of $A_{f}$ in $X$ such that $\left(c_{X}^{*} a\right) \mid N=0$. Thus $c_{X}^{*} a=j^{*} a^{\prime}$, where $a^{\prime} \in H_{G}^{*}(X, N)$ and $j: X \rightarrow(X, N)$ is the inclusion. Let $b \in \operatorname{Ind}^{G}(W-\tilde{W})$. Since we have an equivariant map $X-A_{f} \rightarrow W-\tilde{W}$, we have by (2.3) that $\operatorname{Ind}^{G}(W-\tilde{W}) \subset \operatorname{Ind}^{G}\left(X-A_{f}\right)$. Hence $c_{X}^{*} b \in \operatorname{Ind}^{G}\left(X-A_{f}\right)$, that is, $c_{X}^{*} b \mid\left(X-A_{f}\right)=0$. Thus $c_{X}^{*} b=j^{*} b^{\prime}$, where $b^{\prime} \in H_{G}^{*}\left(X, X-A_{f}\right)$ and $j: X \rightarrow\left(X, X-A_{f}\right)$ is the inclusion. It follows that $c_{X}^{*}(a b)=$ $\left(c_{X}^{*} a\right)\left(c_{X}^{*} b\right)=\left(j^{*} a^{\prime}\right)\left(j^{*} b^{\prime}\right)=j^{*}\left(a^{\prime} b^{\prime}\right)=0$. Therefore $a b \in \operatorname{Ind}^{G} X$.

\section{The cohomology of grassmannians}

Let $V_{m}=V_{m}\left(\mathbb{R}^{\infty}\right)$ denote the Stiefel manifold of orthonormal $m$-frames in $\mathbb{R}^{\infty}$. If $O(m)$ acts on $V_{m}$ in the standard way (by the right multiplication) then the orbit space of the action is the infinite Grassmann space $G_{m}=G_{m}\left(\mathbb{R}^{\infty}\right)$ of $m$-dimensional subspaces of $\mathbb{R}^{\infty}$ and the orbit map $V_{m} \rightarrow G_{m}$ is a classifying bundle for $O(m)$. Thus $H_{0(m)}^{*} V_{m} \cong H^{*} G_{m}$. The cohomology of $G_{m}$ (with coefficients in $\mathbb{Z}_{2}$ ) is a polynomial algebra $\mathbb{Z}_{2}\left[w_{1}, \ldots, w_{m}\right]$ freely generated by the Stiefel-Whitney classes $w_{i} \in H^{i} G_{m}$ of the standard $m$-plane bundle associated to the principal bundle $V_{m} \rightarrow G_{m}$.

The orbit space of the standard free action (right multiplication) of $O(m)$ on the Stiefel manifold $V_{m}\left(\mathbb{R}^{m+n}\right)$ is the real Grassmann manifold $G_{m}\left(\mathbb{R}^{m+n}\right)$ of $m$-dimensional subspaces of $\mathbb{R}^{m+n}$. Thus $H_{O(m)}^{*} V_{m}\left(\mathbb{R}^{m+n}\right) \cong H^{*} G_{m}\left(\mathbb{R}^{m+n}\right)$. There exist two quite different descriptions of the cohomology of $G_{m}\left(\mathbb{R}^{m+n}\right)$. On the one hand, Chern [2] gave a description of the cohomology ring $H^{*} G_{m}\left(\mathbb{R}^{m+n}\right)$ by means of a specific cellular decomposition of the Grassmann manifolds constructed by Ehresmann [3] which, in turn was based on the work of Schubert [15]. By letting $n \rightarrow \infty$, one obtains a decomposition of the infinite Grassmannian $G_{m}=G_{m}\left(\mathbb{R}^{\infty}\right)$. In this decomposition of $G_{m}$ evey cell represents a free generator of the cohomology group in the respective dimension, a monomial in the Stiefel-Whitney classes. On the other hand, the cohomology of $G_{m}\left(\mathbb{R}^{m+n}\right)$ was described in Borel's thesis [1] as a quotient of the polynomial ring on the universal Stiefel-Whitney classes and their duals. A pleasing exposition of the first approach is given in Milnor [13]; compare also Hiller [8].

We can write the total Stiefel-Whitney class as a formal series $w=1+w_{1}+w_{2}+\cdots$ and define the total dual class $\bar{w}=1+\bar{w}_{1}+\bar{w}_{2}+\cdots$ as the formal inverse of $w$, i.e., by the relation

$$
w \bar{w}=1
$$

(compare $[13, \S 4]$ ). Relation (3.1) can be used to express the dual classes $\bar{w}_{i}$ 's in terms of $w_{1}, \ldots, w_{m}$. It contains a countable number of relations, one in each positive dimension.

Definition 3.2. Let $J(m, n)$ denote the ideal in $\mathbb{Z}_{2}\left[w_{1}, \ldots, w_{m}\right]$ generated by $\bar{w}_{1+n}, \ldots, \bar{w}_{m+n}$ expressed as polynomials in $w_{1}, \ldots, w_{m}$ by using (3.1). 
Theorem 3.3. $J(m, n)$ is the $O(m)$-index of $V_{m}\left(\mathbb{R}^{m+n}\right)$.

Borel [1] showed that the albegra $H_{0(m)}^{*} V_{m} \cong H_{0(m)}^{*}(*) \cong H^{*} G_{m}$ is isomorphic to the quotient algebra $\mathbb{Z}_{2}\left[w_{1}, \ldots, w_{m}, \bar{w}_{1}, \bar{w}_{2}, \ldots\right] / I(m)$, where $I(m)$ is the ideal in $\mathbb{Z}_{2}\left[w_{1}, \ldots, w_{m}, \bar{w}_{1}, \bar{w}_{2}, \ldots\right]$ generated by the homogeneous terms of $w \bar{w}$ of positive dimension. As shown by Borel, it follows that

$$
H^{*} G_{m}\left(\mathbb{R}^{m+n}\right) \cong \mathbb{Z}_{2}\left[w_{1}, \ldots, w_{m}, \bar{w}_{1}, \ldots, \bar{w}_{n}\right] / I(m, n),
$$

where $I(m, n)$ is the ideal in the polynomial algebra $\mathbb{Z}_{2}\left[w_{1}, \ldots, w_{m}, \bar{w}_{1}, \ldots, \bar{w}_{m}\right]$ generated by the $m+n$ terms of $\left(1+w_{1}+\cdots+w_{m}\right)\left(1+\bar{w}_{1}+\cdots+\bar{w}_{n}\right)$ of positive dimension. The relations corresponding to the first homogeneous terms of the latter product (in dimensions $1, \ldots, n$ ) yield $n$ equations

$$
w_{k}+w_{k-1} \bar{w}_{1}+\cdots+w_{1} \bar{w}_{k-1}+\bar{w}_{k}=0, k=1, \ldots, n
$$

which can be solved recursively for $\bar{w}_{1}, \ldots, \bar{w}_{n}$ (see $[13$, p. 40]). Substituting the resulting formulas to the remaining $m$ homogeneous terms of the product (in dimensions $1+n, \ldots, m+n)$ we obtain the ideal $J(m, n)$. Thus $H^{*} G_{m}\left(\mathbb{R}^{m+n}\right) \cong \mathbb{Z}_{2}\left[w_{1}, \ldots, w_{m}\right] / J(m, n)$. The $O(m)$-index of $V_{m}\left(\mathbb{R}^{m+n}\right)$ is $\operatorname{Ind}^{O(m)} V_{m}\left(\mathbb{R}^{m+n}\right)=\left(\operatorname{Ker}\left(c^{*}: H_{O(m)}^{*}(*) \rightarrow H_{O(m)}^{*} V_{m}\left(\mathbb{R}^{m+n}\right)\right)\right.$. Since the action of $O(m)$ on $V_{m}\left(\mathbb{R}^{m+n}\right)$ is free, $H_{O(m)}^{*} V_{m}\left(\mathbb{R}^{m+n}\right) \cong H^{*} G_{m}\left(\mathbb{R}^{m+n}\right)$ and, under this isomorphism, the kernel of $c^{*}$ coincides with the kernel of $\varphi^{*}: H^{*} G_{m} \rightarrow H^{*} G_{m}\left(\mathbb{R}^{m+n}\right)$, where $\varphi$ is a classifying map for $V_{m}\left(\mathbb{R}^{m+n}\right)$ (compare [11, (5.1)]); for instance, $\varphi$ can be in the inclusion $G_{m}\left(\mathbb{R}^{m+n}\right) \rightarrow G_{m}$. Thus $\varphi^{*}$ corresponds to the quotient map $\mathbb{Z}_{2}\left[w_{1}, \ldots, w_{m}\right] \rightarrow \mathbb{Z}_{2}\left[w_{1}, \ldots, w_{m}\right] / J(m, n)$ whose kernel is $J(m, n)$.

Let $J(m, n)$, denote the $r$-dimensional component of the ideal $J(m, n)$. Consider the map

$$
\gamma(r, n): H^{r-n-1} G_{m} \oplus \cdots \oplus H^{r-n-m} G_{m} \rightarrow H^{r} G_{m}
$$

defined by $\left(x_{1}, \ldots, x_{m}\right) \rightarrow \bar{w}_{1+n} x_{1}+\cdots+\bar{w}_{m+n} x_{m}$.

Lemma 3.5. $J(m, n)_{r}=\operatorname{Im} \gamma(r, n)$.

For the proof it is enough to observe that every element in $J(m, n)_{r}$ can be written as an element of $\operatorname{Im} \gamma(r, n)$ by grouping similar terms with respect to $\bar{w}_{1+n}, \ldots, \bar{w}_{m+n}$.

If $r \leqq n$ then $J(m, n)_{r}=0$ and the inclusion $G_{m}\left(\mathbb{R}^{m+n}\right) \subset G_{m}$ induces an isomorphism $H^{r} G_{m} \cong H^{r} G_{m}\left(\mathbb{R}^{m+n}\right)$. Thus for $r \leqq n, H^{r} G_{m}\left(\mathbb{R}^{m+n}\right)$ is additively generated by all monomials $w_{1}^{q_{1}}, \ldots, w_{m}^{q_{m}}$ of a total degree $q_{1}+2 q_{2}+\cdots+m q_{m}=r$. In this range of $r$, the rank of $H^{r} G_{m}\left(\mathbb{R}^{m+n}\right)$ is equal to the number $p_{m}(r)$ of all partitions of $r$ into at most $m$ integers (see $[13$, p. 85]).

Let $p_{m}^{n}(r)$ denote the number of partitions of $r$ into at most $m$ positive integers each of which is $\leqq n$. 
Proposition 3.6. Rank $H^{r} G_{2}\left(\mathbb{R}^{2+n}\right)=p_{2}^{n}(r)$.

Proof. If $r \leqq n$ then this rank is $p_{2}(r)$ which is equal to $p_{2}^{n}(r)$. Suppose $n \leqq r \leqq 2 n$. Since $G_{2}\left(\mathbb{R}^{2+n}\right)$ is a $2 n$-manifold, by the Poincaré Duality, Rank $H^{r} G_{2}\left(\mathbb{R}^{2+n}\right)=p_{2}(2 n-r)=$ $p_{2}^{n}(r)$.

Remark 3.7. For $m=2, p_{2}(r)=[r / 2]+1$.

\section{Maps of Stiefel manifolds}

We return now to our example to Section 1. Thus $X=V_{m}\left(\mathbb{R}^{m+n}\right)$ and $W=\left(\mathbb{R}^{m+k}\right)^{m}$ have the standard (right) action of $O(m), f: X \rightarrow W$ is a map, $\tilde{W}$ is the subset of $W$ consisting of $m$-tuples of vectors in $\mathbb{R}^{m+n}$ which are not linearly independent, $W_{0}=$ $W-\tilde{W}$, and $A_{f}=(\operatorname{Av} f)^{-1} \tilde{W}$. Then the Gram-Schmidt orthogonalization process provides a homotopy equivalence $W_{0} \cong V_{m}\left(\mathbb{R}^{m+k}\right)$. Thus $\operatorname{Ind}^{\mathrm{o(m)}} X=J(m, n)$, Ind ${ }^{\mathrm{o}(m)} W_{0}=$ $J(m, k)$ and by $(2.4)$ we have the following inclusion.

Theorem 4.1. $\quad\left(\operatorname{Ind}^{0(m)} A_{f}\right) \cdot J(m, k) \subset J(m, n)$.

Remark 4.2. If $m=1$ then $X=S^{n}, W=\mathbb{R}^{k+1}, J(m, n)$ is the ideal in $H_{Z_{2}}^{*}(X) \cong H^{*} \mathbb{R} P^{\infty} \cong$ $\mathbb{Z}_{2}\left[w_{1}\right]$ generated by $w_{1}^{n+1}$, and $J(m, k)$ is the ideal generated by $w_{1}^{k+1}$. The index Ind $^{0(1)} A_{f}=\operatorname{Ind}^{\mathbb{Z}_{2}} A_{f}$ is generated by $w_{1}^{j+1}$, for some integer $j$, the classical $\mathbb{Z}_{2}$-index of $A_{f}$. In this case, the inclusion of (4.1) is equivalent to the inequality $j \geqq n-(k+1)$, which is the assertion of the classical Borsuk-Ulam-Yang theorem.

Thus Theorem 4.1 contains information about the size of $A_{f}$ : it asserts that the cohomology ring of $A_{f}$ cannot be too small: its index is bounded above. However, deciding in a particular case which universal cohomology class of $H_{0(m)}^{*}(*)$ survive by not finding themselves in the index of $A_{f}$, can be a non-trivial task. In an effort to extract a more specific information about the size of $A_{f}$, we shall attempt to determine a highest possible dimension where the cohomology of $A_{f} / 0(m)$, or the $O(m)$-cohomology of $A_{f}$, is non-zero. This will be done in the next section in the case $m=2$. In Section 6 we will be able to obtain a better result for the special case when $m=2, n=2^{s}-1$ and $k=n-1$.

\section{The case $m=2$}

We shall keep the notation of Section 1 . Thus for a map $f: V_{2}\left(\mathbb{R}^{n+2}\right) \rightarrow\left(\mathbb{R}^{k+2}\right)^{2}$ we have

$$
\left(\operatorname{Ind}^{0(2)} A_{f}\right) \cdot J(2, k) \subset J(2, n) .
$$

Theorem 5.2. If $k<n$ and $f: V_{2}\left(\mathbb{R}^{n+2}\right) \rightarrow\left(\mathbb{R}^{k+2}\right)^{2}$ is a map then $H^{*}\left(A_{f} / 0(2)\right)$ is non-zero in a dimension at least $2 n-k-2$. 
Corollary 5.3. The covering dimension of $A_{f} / 0(2), \operatorname{dim} A_{f} / 0(2)$, is at least $2 n-k-2$. Furthermore, since the orbit map $A_{f} \rightarrow A_{f} / O(2)$ is a bundle with fibre $O(2), \operatorname{dim} A_{f} \geqq 2 n-$ $k-1$.

Proof of (5.2). According to (3.6), Rank $H^{2 n} G_{2}\left(\mathbb{R}^{n+2}\right)=p_{2}^{n}(2 n)=1$; this also follows from the fact that $G_{2}\left(\mathbb{R}^{n+2}\right)$ is a $2 n$-dimensional manifold. Let $v_{2 n} \in H^{2 n} G_{2}\left(\mathbb{R}^{n+2}\right) \cong \mathbb{Z}_{2}$ be the non-zero class (in fact, $v_{2 n}$ is the image under the natural map $H^{2 n} G_{2} \rightarrow H^{2 n} G_{2}\left(\mathbb{R}^{n+2}\right)$ of $w_{2}^{n} \in H^{2 n} G_{2}$; see [8, Lemma (1.2)]. Thus the $2 n$-component $J(2, n)_{2 n}$ of the index does not contain the entire $H^{2 n} G_{2}$. This means that there is a class (in this case, it is $w_{2}^{n}$ ) in $H^{2 n} G_{2}$ which is not in $J(2, n)_{2 n}$.

On the other hand, also because $G_{2}\left(\mathbb{R}^{k+2}\right)$ is a $2 k$-dimensional manifold, $H^{2 n} G_{2}\left(\mathbb{R}^{k+2}\right)=0$ for $k<n$. Therefore the $2 n$-component $J(2, k)_{2 n}=\operatorname{Im} \gamma(2 n, k)$ of the index (cf. (3.5)) contains the entire cohomology module $H^{2 n} G_{2}$. This means that the map

$$
\gamma(2 n, k): H^{2 n-k-1} G_{2} \oplus H^{2 n-k-2} G_{2} \rightarrow H^{2 n} G_{2}
$$

is surjective. It follows that there exists a pair $(x, y) \in H^{2 n-k-1} G_{2} \oplus H^{2 n-k-2} G_{2}$ such that $\gamma(2 n, k)(x, y) \notin J(2, n)$. By the definition of $\gamma, \bar{w}_{k+1} x+\bar{w}_{k+2} y \notin J(2, n)$. But $\bar{w}_{k+1}$ and $\bar{w}_{k+2}$ are in $J(2, k)=\operatorname{Ind}^{0(2)} V_{2}\left(\mathbb{R}^{k+2}\right)$, hence it follows (5.1) implies that either $x \notin \operatorname{Ind}^{0(2)} A_{f}$ or $y \notin \operatorname{Ind}^{0(2)} A_{f}$. This means that either the image of $x$ or the image of $y$ is a non-zero class in $H_{0(2)}^{*} A_{f} \cong H^{*}\left(A_{f} / 0(2)\right)$.

\section{The case $m=2$ and $n=2^{s}-1$}

In the case $m=2, n=2^{s}-1$ and $k=n-1$ the result of (5.2) can be improved.

Theorem 6.1. If $n=2^{s}-1$ and $f: V_{2}\left(\mathbb{R}^{n+2}\right) \rightarrow\left(\mathbb{R}^{n+1}\right)^{2}$ is a map then $H^{n}\left(A_{f} / O(2)\right) \neq 0$. Just as in (5.3) we have

Corollary 6.2. The covering dimension $\operatorname{dim} A_{f} / 0(2) \geqq n$; hence $\operatorname{dim} A_{f} \geqq n+1$.

\section{Lemma 6.3.}

$$
\frac{\left(2^{s}-i-1\right)}{\left(2^{s}-2 i-1\right) ! i !} \equiv 0 \bmod 2, \text { for } i>0
$$

Proof.

$$
\begin{aligned}
\frac{\left(2^{s}-i-1\right) !}{\left(2^{s}-2 i-1\right) ! i !} & =\frac{\left(2^{s}-2 i\right)\left(2^{s}-2 i+1\right) \ldots\left(2^{s}-i-1\right)}{i !} \\
& \equiv \frac{(2 i)(2 i-1) \ldots(i+1)}{i !} \bmod 2 \\
& =\frac{(2 i) !}{i ! i !}=\frac{(2 i-1) !}{(i-1) ! i !}+\frac{(2 i-1) !}{i !(i-1) !} \equiv 0 \bmod 2,
\end{aligned}
$$


because the binomial coefficients $(a+b) ! / a ! b$ ! satisfy the relation

$$
\frac{(a+b) !}{a ! b !}=\frac{(a+b-1) !}{(a-1) ! b !}+\frac{(a+b-1) !}{a !(b-1) !} .
$$

Proof of 6.1. Relation (6.1) for $k=n-1$ now reads

$$
\left(\text { Ind }^{0(2)} A_{f}\right) \cdot J(2, n-1) \subset J(2, n) .
$$

The ideal $J(2, n-1)$ is generated by $\bar{w}_{n}$ and $\bar{w}_{n+1}$, and $J(2, n)$ is generated by $\bar{w}_{n+1}$ and $\bar{w}_{n+2}$ in $\mathbb{Z}_{2}\left[w_{1}, w_{2}\right]$. The dual class $\bar{w}_{n}$ is a polynomial consisting of the terms of

$$
\bar{w}=\left(1+\left(w_{1}+w_{2}\right)\right)^{-1}=1+\left(w_{1}+w_{2}\right)+\left(w_{1}+w_{2}\right)^{2}+\cdots
$$

of total degree $n=2^{s}-1$. Thus

$$
\bar{w}_{n}=\sum_{i=0}^{2^{s-1}-1} \frac{\left(2^{s}-i-1\right) !}{\left(2^{s}-2 i-1\right) ! i !} w_{1}^{2 s-2 i-1} w_{2}^{i} .
$$

By (6.3), all the coefficients in this polynomial for $i>0$ are zero, and for $i=0$, the coefficient of $w_{1}^{2 s-1}$, is 1 . Thus $\bar{w}_{n}=w_{1}^{2 s-1}=w_{1}^{n}$. Therefore $w_{1}^{n} \in J(2, n-1)$. We claim, however, that $w_{1}^{n} \notin \operatorname{Ind}^{0(2)} A_{f}$. For, if $w_{1}^{n}$ were in $\operatorname{Ind}^{0(2)} A_{f}$, relation (7.4) would imply that $w_{1}^{n} \cdot w_{1}^{n}=w_{1}^{2 n}$ would belong to $J(2, n)$. However, it was proved by Hiller [8] that $w_{1}^{2 n}=w_{1}^{2 s+1-2}$ is not zero in $H^{*} G_{2}\left(\mathbb{R}^{n+2}\right)$; that is, $w_{1}^{2 n} \notin J(2, n)$.

This completes the proof.

Acknowledgement. The author gratefully acknowledges a support received from the "Deutsche Forschungsgemeinschaft" at the Ludwig-Maximilian University in Munich in $1985-86$ during the preparation of this paper.

\section{REFERENCES}

1. A. Borel, La cohomologie mod 2 de certain espaces homogènes, Comm. Math. Helv. 27 1953), 165-197.

2. S. Chern, On the multiplication in the characteristic ring of a sphere bundle, Ann. of Math. 49 (1948), 362-372.

3. C. Ehresmann, Sur la topologie de certains espaces homogènes, Ann. of Math. 35 (1934), 396-443.

4. E. R. Fadell, S. Y. Husseinl and P. H. Rabinowitz, Borsuk-Ulam theorems for arbitrary $S^{1}$ actions and applications, Trans. Amer. Math. Soc. 274 (1982), 345-360.

5. E. R. Fadell, S. Y. Husseini, Index theory for G-bundle pairs with applications to BorsukUlam type theorems for $G$-sphere bundles, preprint.

6. E. R. FADELl and S. HusseInI, Relative cohomological index theories, preprint.

7. E. R. F.ADELl and S. Y. HusseINI; Cohomological Index Theory with Applications to Critical Point Theory and Borsuk-Ulam Theorems. (A series of lectures, NATO Advanced Study Institute on "Variational Methods in Nonlinear Problems," Montreal, July 1986.) 
8. H. L. Hiller, On the cohomology of real Grassmannians, Trans. Amer. Math. Soc. 257 (1980), 521-533.

9. J. JAworowski, A continuous version of the Borsuk-Ulam theory, Proc. Amer. Math. Soc. 82 (1981), 112-114.

10. J. JAworowskI, Fibre Preserving Maps of Sphere Bundles in Vector Space Bundles (Proc. of the Fixed Point Theory Workshop: Sherbrooke, 1980; Lecture Notes in Mathematics, 886, Springer-Verlag, 1981), 154-162.

11. J. Jaworowskı, The set of balanced orbits of maps of $S^{1}$ and $S^{3}$ actions, Proc. Amer. Math. Soc. 98 (1986), 158-162.

12. J. JAWORowskı, Balanced Orbits for Fibre Preserving Maps of $S^{1}$ and $S^{3}$ Actions, (Proc. of the Transformation Groups Symposium, Poznan 1985; Lecture Notes in Mathematics, 1217, Springer-Verlag, 143-150.

13. J. W. Milnor and J. D. Stasheff, Characteristic Classes, Ann. of Math. Stud. 76 (1974).

14. M. NAKaOKa, Equivalent point theorems for fibre-preserving maps, Osaka J. Math. 21 (1984), 209-815.

15. H. Schubert, Kalkül der abzählenden Geometrie (Teubner, Leipzig, 1879).

16. H. Steinlein, Borsuk's theorem and its generalizations and applications: A survey, Sem. Math. Sup. 95 (1985), 166-235.

17. C. T. YANG, On theorems of Borsuk-Ulam, Kakutani-Yamabe-Yujobo and Dyson. II, Ann. of Math. (2) (1955), 166-283.

Department of Mathematics

INDIANA UNIVERSITY

BLOOMINGTON, IN 47405 